\title{
Graphene Quantum Dots: Beyond a Dirac Billiard
}

\author{
Florian Libisch ${ }^{1}$, Christoph Stampfer ${ }^{2}$, and Joachim Burgdörfer ${ }^{1}$ \\ ${ }^{1}$ Institute for Theoretical Physics, Vienna University of Technology \\ Wiedner Hauptstraße 8-10/136, A-1040 Vienna, Austria, European Union \\ ${ }^{2}$ Solid State Physics Laboratory, ETH Zurich, 8093 Zurich, Switzerland
}

(Dated: October 25, 2018)

\begin{abstract}
We present realistic simulations of quantum confinement effects in ballistic graphene quantum dots with linear dimensions of 10 to $40 \mathrm{~nm}$. We determine wavefunctions and energy level statistics in the presence of disorder resulting from edge roughness, charge impurities, or short-ranged scatterers. Marked deviations from a simple Dirac billiard for massless fermions are found. We find a remarkably stable dependence of the nearest-neighbor level spacing on edge roughness suggesting that the roughness of fabricated devices can be potentially characterized by the distribution of measured Coulomb blockade peaks.
\end{abstract}

PACS numbers: 73.22.Dj, 81.05.Uw, 05.45.Mt

Graphene [1, 2], the first true two-dimensional (2D) solid, is attracting considerable attention, mostly due to unique dynamics of electrons near the Fermi energy which closely mimics that of a massless Dirac Hamiltonian [see Fig.1(a)]. Moreover, the double cone structure near the $K$ and $K^{\prime}$ points of the sublattices in reciprocal space gives rise to a near "pseudospin" degeneracy, suggesting an analog of Dirac four spinors. Envisioned applications range from high-mobility nanoelectronics [3], spin-qubits in graphene quantum dots [4] and the creation of "neutrino" billiards 5, 6]. Dirac (including neutrino) billiards receive growing interest as a complement to classical and quantum (Schrödinger) billiards, which have taken central stage in studies elucidating the quantum-to-classical crossover in both regular and chaotic devices. Additionally, spin coherence times in graphene are expected to be very long due to weak spin-orbit and hyperfine couplings [7, 8] making graphene quantum dots promising for future spin based quantum computation [4]. However, confining electrons in graphene is a challenge, due to the gap-less electronic structure and the Klein tunneling paradox [9, 10]. This difficulty has recently been overcome by structuring $2 \mathrm{D}$ graphene and quantum mechanical confinement effects have been observed in nanoribbons 11, 12, 13 , interference devices [14], single electron transistors 15, 16] and graphene quantum billiards [6].

In the following we present a realistic simulation for the single particle spectrum of graphene quantum dots (i.e. billiards) by explicitly considering rough edges and disorder. This work was motivated by recent advances in fabricating dots with linear dimension $d$ ranging from a few hundred $\mathrm{nm}$ down to about $40 \mathrm{~nm}$, and determining their nearest neighbor energy level spacing distribution [6, 16]. We analyze dot wavefunctions, the density of states (DOS) and the nearest neighbor spacing distribution (NNSD). We address the question to what extent the electron spectra now experimentally accessible via measurements of Coulomb blockade peaks reveal information
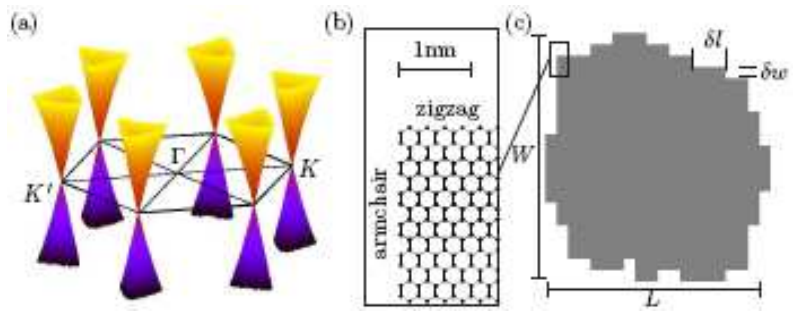

FIG. 1: (color online) (a) Graphene dispersion near $K$ and $K^{\prime}$ points of the infinitely extended sheet. (b) Rectangular segment of a graphene flake, vertical edge armchair, horizontal edge zig-zag terminated. (c) (Approximately) rectangular quantum dot with rough edges.

on the roughness and size of the graphene quantum dot. To put it provocatively: Can one "hear" the rugged shape of a drum if it is made of a graphene flake?

We investigate graphene dots with linear dimensions between 10 and $40 \mathrm{~nm}$, in line with currently fabricated devices [6]. The shape is chosen regular in the absence of edge roughness. One motivation of this choice was the remarkable result [5] that a Dirac neutrino billiard, in sharp contrast to a Schrödinger billiard, would feature chaotic dynamics. We consider a ballistic dot, thereby neglecting inelastic scattering. This is justified as the inelastic mean free path $\lambda_{i l}$ found in experiment exceeds the linear dimension $d=\sqrt{4 L W / \pi}$ of the dot, $d \leq 40 \mathrm{~nm}$ $\ll \lambda_{e l} \approx 400 \mathrm{~nm}[1]$. Our simulation allows for the inclusion of disorder through (i) edge roughness [see Fig. 1(c)], (ii) short-range disorder due to point defects in the interior, and (iii) long-range screened Coulomb distortion due to charge deposition. Rough edges are simulated by modulating the boundary of the dot by steps of height $\pm \delta w$ and length $\pm \delta l$ randomly chosen from the interval $[0, \Delta W], \Delta W \ll d$. We refer to $\Delta W$ as the amplitude of edge roughness which varies between $0.3 \mathrm{~nm}$ (weak disorder) and $2 \mathrm{~nm}$ (strong disorder). The resulting piecewise straight edge features alternating zig-zag and armchair sections [see Figs. 1(b,c)]. We describe impurities 
and defects in the flake by positioning either long range $\left(V(\mathbf{r})=V_{0} e^{-\alpha\left|\mathbf{r}-\mathbf{r}_{0}\right|}\right)$ or short range $\left(V(\mathbf{r})=\delta\left(\mathbf{r}-\mathbf{r}_{0}\right)\right)$ scatterers at randomly selected lattice sites $\mathbf{r}_{0}$. We use an impurity density $n_{i}<1.8 \cdot 10^{-3}$ impurities/carbon (10 to 100 defects per flake), as estimated by recent work [17.

The spectrum of the graphene quantum dots is determined employing a Lanczos algorithm [18] giving the 500 eigenstates closest to the Fermi edge. The graphene flake is described by a third nearest neighbor tight-binding approximation to correctly reproduce the graphene bandstructure [19]. The modified C-C bond length at the flake boundary is accounted for by increasing nearestneighbor coupling to the outmost carbon atoms by $12 \%$ in accordance with recent ab-initio density functional calculations 20]. Our ensemble averages for the DOS $\langle\rho\rangle_{\xi}=\left\langle\sum_{i} \delta\left(E-E_{i}\right)\right\rangle_{\xi}$ encompass typically 5000 disorder realizations $\xi$.

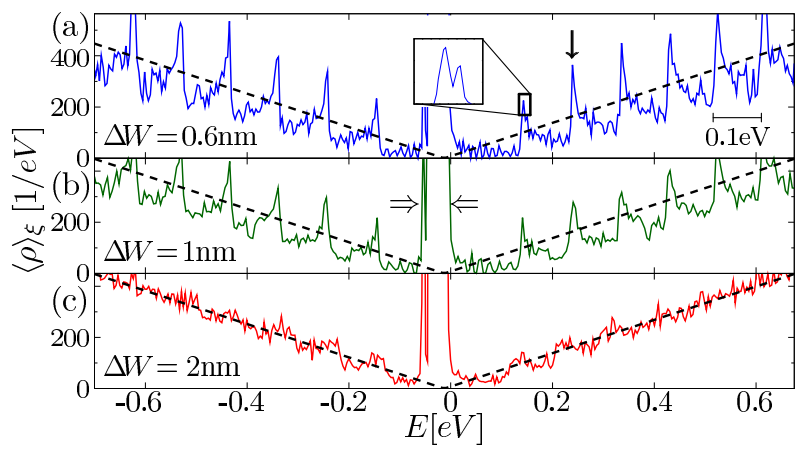

FIG. 2: (color online) Ensemble-averaged density of states $\langle\rho(E)\rangle_{\xi}$ of graphene quantum dots with increasing edge roughness, see different values for $\Delta W$ in the subfigures. The size of all devices is equal, $d=20 \mathrm{~nm}$ (20.000 atoms), their width $W=16 \mathrm{~nm}$. Dashed lines indicate the averaged linear DOS for Dirac billiards [see Eq. (1)]. The inset shows the $K-K^{\prime}$ splitting of $12 \mathrm{meV}$.

The linear dispersion relation of a massless Dirac particle implies a DOS linear in $\varepsilon$,

$$
\rho(\varepsilon)=\frac{1}{2\left(\hbar v_{\mathrm{F}}\right)^{2}} d^{2}|\varepsilon|,
$$

where $d=\sqrt{4 W L / \pi}$ is the effective diameter of a dot with area $W L$ and $\varepsilon$ is measured relative to the conical intersection [Fig. 1(a)] assumed to coincide with the Fermi edge. The simulated DOS for the quantum dots display marked deviations from Eq. (11). For weak disorder pronounced size quantization peaks appear [see e.g. vertical arrow in Fig. 2(a)]. Their positions are determined by the smallest linear dimension of the flake [21]. We have investigated both cases $W<L(W>L)$, and find the same qualitative behavior. In the following, we assume $W<L$. Note that width $(W)$ and length $(L)$ of the rectangular flake are not strictly equivalent as the vertical boundary features an arm-chair border while the horizontal forms a zig-zag border. The distance between the quantization peaks is $\Delta E=\hbar v_{\mathrm{F}} \pi / W \approx 1.5 / W[\mathrm{eV}]$, width $W$ given in $\mathrm{nm}$. This yields an analytic prediction for the energy separation of $0.1 \mathrm{eV}$ for the peaks in Fig. 2. Weak disorder, i.e. small edge roughness can induce coupling between the cones at $K$ and $K^{\prime}$ [Fig. 1(a)]. This manifests itself by a fine structure of size quantization by lifting the degeneracy [inset in Fig. 2(a)]. The quantum confinement peaks in the graphene dot are enhanced compared to a corresponding Schrödinger billiard of the same geometry in part because of both the altered dispersion relation and the additional degeneracy. Strong disorder smears out size quantization patterns and the DOS begins to resemble that of a zero-mass Dirac fermion in free space (Eq. 11). Only when the edge roughness can be limited to the sub-nanometer scale, quantized conductance in graphene nanoribbons persists [13].

Even in the limit of strong disorder, the prominent peak in the DOS near the Fermi edge remains unchanged [see horizontal arrows in Fig. 2(b)]. A direct look at the wavefunction [Fig. 3(a)] reveals its origin: A large number of strongly (Anderson-)localized states at the edges of the graphene flake. Each eigenstate features a nonvanishing amplitude only at a few, not always spatially connected carbon atoms, with a decay length into the bulk of typically $0.5 \mathrm{~nm}$ [see left arrow in Fig. 3(a)]. We find that the eigenenergies of these states are extremely sensitive to the site energies at the corresponding lattice sites. This agrees well with the experimental observations of sharp resonance in the electron transport through graphene constrictions [15].

Delocalized states contributing to the size quantization peaks show pronounced features well beyond the simple picture of a confined zero-mass Dirac particle. While the transverse quantization resembles that of a conventional conductor, the interference pattern in the electron probability density [Fig. 3(b)] results from the simultaneous presence of multiple wavelength scales for the cone near the $K$ point (unlike wavefunctions near the $\Gamma$ point), in $\mathbf{k}=\left(k_{0}+v_{\mathrm{F}} \pi / L, v_{\mathrm{F}} \pi / W\right)$ : Parallel to armchair edges (i.e. in vertical direction in Fig. 3), the wavelength is of the order of twice the width of the ribbon-like dot $\approx 32 \mathrm{~nm}$. Parallel to zigzag edges, (i.e. in horizontal direction in Fig. (3) the wave oscillations are much shorter with a typical wavelength of $0.7 \mathrm{~nm}$ [see Fig. 3(b)] resulting from beating (frequency ratio 3:2) between lattice periodicity $a=0.24 \mathrm{~nm}$ and the characteristic wavelength $\lambda_{0}=2 \pi / k_{0} \approx 0.37 \mathrm{~nm}$, where $k_{0} \gg v_{\mathrm{F}} \pi / L$ is the distance between $\Gamma$ and $K$ point in reciprocal space [see Fig. 1(a)]. We find beating patterns with this characteristic length scale to be universally present in all delocalized states, even in the presence of long-range disorder. Only because of the sub-nanometer length scale of $\lambda_{0}$ at the $K$ point is the graphene dot sensitive to edge roughness and disorder on a length scale of a few nanometers, in contrast to a Dirac cone at the $\Gamma$ point. The reason for the $K$-point $\left(k_{0}, 0\right)$ [as opposed to those at 60 and 


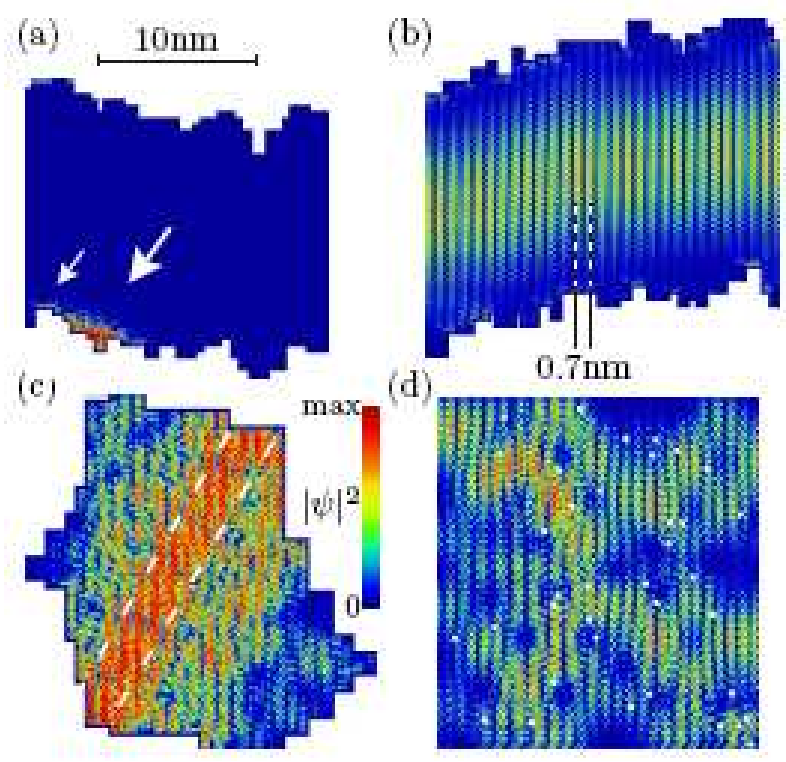

FIG. 3: (color online) Eigenstates of graphene quantum dots with dot area of about $225 \mathrm{~nm}^{2}$ (13.000 carbon atoms). Eigenenergies are (a) $65 \mathrm{meV}$ (b) $220 \mathrm{meV}$ (c) $650 \mathrm{meV}$ (d) $1020 \mathrm{meV}$ respectively. The edge roughness parameter $\Delta W=1 n m$ in (a-c) while $n_{i}=0.005$ in (d) (White dots mark the center of long-range impurities).

120 degrees, $\left.\frac{1}{2}\left(k_{0}, \pm \sqrt{3} k_{0}\right)\right]$, to appear in the eigenstate shown is the orientation of the zigzag (armchair) edges in the flake parallel (orthogonal) to the $\left(k_{0}, 0\right)$ direction. However, all three directions appear for higher transverse quantum numbers, resulting in enhancements along the three zigzag-directions of the lattice [i.e. horizontal, 60 and 120 degrees, see dashed lines in Fig. 3(c)]. As a consequence, eigenstates feature a 2D hole ("swiss-cheese") pattern emerging from the interference of plane waves rotated by 60 degrees relative to each other [see Fig. 3 (d)].

In order to delineate the influence of disorder and edge roughness on the energy level statistics, we have determined the NNSD, $P(\Delta E)$, i.e. the probability that the energy difference between two adjacent energy levels is $\Delta E$, for different amplitudes $\Delta W$ of roughness. Within the framework of quantum dynamics of Schrödinger billiards, $P(\Delta E)$ follows a Poisson distribution for separable (classically regular) shapes while it should display a Wigner-Dyson (or GOE) distribution for irregularly shaped (classically chaotic) billiards. In contrast, even rectangular shaped Dirac neutrino billiards have been shown to feature a GUE distribution because of the broken time reversal symmetry due to chirality [5]. After spectral unfolding $\left[s=\bar{\rho}\left(\varepsilon_{i}\right)\right.$, with $\langle P(s)\rangle=1$ and $\langle s P(s)\rangle=1]$ we find for the ideal rectangular graphene $\operatorname{dot}(\Delta W=0)$ a near-perfect Poisson distribution [see Fig. 4(a)]. By gradually increasing either the edge roughness or the defect concentration $n_{i}$, the distribution smoothly evolves into a Wigner-Dyson like statistics [see

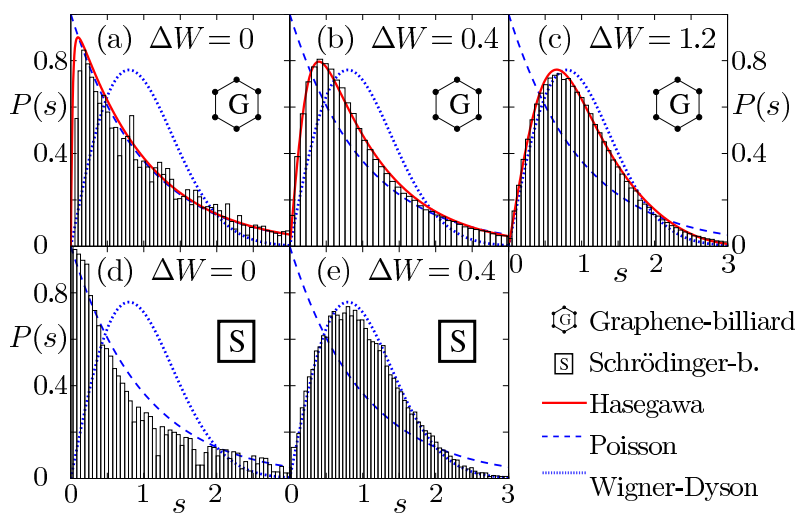

FIG. 4: (color online) NNSD $P(s)$ of different billiards: Rectangular graphene flake with (a) smooth edges $(\beta=0.07)$. (b) finite edge roughness $\Delta W=0.5 \mathrm{~nm}(\beta=0.4)$, (c) $\Delta W=1 \mathrm{~nm}(\beta=2.5)$. (d,e) Schrödinger billiards with same edge roughness as $(a, b)$. The solid red curve shows fits to the Hasegawa distribution. Dashed (dotted) lines show a Wigner-Dyson (Poisson) statistic as guide to the eye.

Fig. 4(b,c)]. Clearly, such a behavior reflects the conservation of time-reversal symmetry in graphene quantum dots. Among the distribution functions suggested for the transition regime for classically mixed phase space [22, 23, 24, 25, 26], the best fit for the disorder parameters and geometries investigated was achieved for the two-parameter Hasegawa distribution [27]

$$
P_{H}(s ; \alpha, \beta)=N \frac{\rho s e^{-\rho s-(\alpha \rho s)^{2} / 2}}{\sqrt{\rho^{2} s^{2} e^{-\alpha^{2} \rho^{2} s^{2}}+\beta^{2} e^{-2 \rho s}}},
$$

where $\rho$ and $N$ are determined by the normalization conditions $\left\langle P_{H}\right\rangle=\left\langle s P_{H}\right\rangle=1$ [27]. While the control parameter $\beta$ describes the transition from Poissonian $(\beta=0)$ to Wigner-Dyson statistics $(\beta \rightarrow \infty), \alpha$ is a systemspecific constant. Indeed, we find $\alpha=0.75$ to correctly reproduce our numerically obtained NNSD for different values of both edge roughness as well as scatterers [see Fig. $4(\mathrm{a}-\mathrm{c})$ ). A strong edge roughness of $2 \mathrm{~nm}$ (or impurity concentration $n_{i}=5 \cdot 10^{-3}$ ) is required to reach the completely chaotic limit, i.e. a Wigner-Dyson NNSD statistics. Remarkably, for moderate values of the edge roughness amplitude $(\Delta W=0.6 \mathrm{~nm})$ a Schrödinger billiard and a graphene billiard of the same geometry display a markedly different NNSD (Fig. 4): While the Schrödinger billiard has already reached the WignerDyson limit $(\beta \rightarrow \infty)$, for the graphene the NNSD still is closer to the Poisson limit, pointing to the unique spectral properties of graphene. The quasi-regular dynamics in graphene is more stable against disorder than in corresponding Schrödinger billiards. The origin of this, at first glance, surprising finding is closely related to the electronic structure of graphene at the $K$ point [Fig. 1(a)]. In a classical rectangular ballistic billiard with only rectangular edges along the armchair or zig-zag direction 

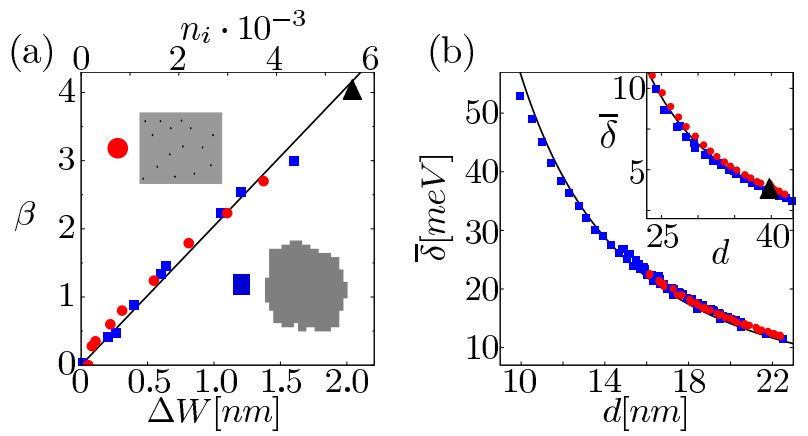

FIG. 5: (color online) (a) Dependence of the control parameter $\beta$ for the transition from a Poisson to a Wigner-Dyson distribution (Eq. 2) on the edge roughness amplitude $\Delta W$ or the defect density $n_{i}$. (b) Dependence of rescaled mean-level spacing $\bar{\delta}=\left\langle\Delta E \cdot E / E_{0}\right\rangle \mathrm{meV}$, where $E_{0}$ was taken at $100 \mathrm{meV}$, on the size of the quantum dot. Triangles represent experimental data, rescaled by $\Delta E \rightarrow\left(\Delta E-E_{C}\right) \frac{E}{E_{0}}=(\Delta E-5) \frac{50}{100}$ to take into account the charging energy $E_{C}$ and the energy dependence of the DOS. [6].

an additional constant of motion, the magnitude of the linear momentum $|\mathbf{k}|$, exists. Such billiards are therefore classically regular irrespective of the number or size of the edges. By contrast, due to the larger deBroglie wavelength of Schrödinger billiards with wavenumbers near the $\Gamma$ point cannot resolve the exactly rectangularly shaped edges, and thus mimics chaotic dynamics.

Of potential technological significance is the dependence of the NNSD on the disorder in graphene billiards. For all three classes of disorder we consider (edge roughness, short and long range disorder) we find a linear relation between the NNSD parameter $\beta$ and the edge roughness amplitude, $\beta \approx 2 \Delta W$, and between $\beta$ and the defect density, $\beta \approx 0.7 n_{i}$ [Fig. [5(a)]. As $\beta$ can be obtained with high precision from a fit to $P(s)$, the edge roughness or defect density can be deduced from the NNSD, if the distribution is Poisson-like in the limit $\Delta W=0\left(n_{i}=0\right)$. We suggest that this dependence could be used to estimate the disorder in experimentally realized regularly shaped ballistic graphene quantum dots. Numerically, we find $\beta \approx 4$ in a fit to data from recent experimental investigations of a 40nm graphene billiard [6], corresponding to an effective roughness $\Delta W_{e} \approx 2 \mathrm{~nm}$, or an effective defect rate $n_{i} \approx 5.5 \cdot 10^{-3}$ (see black triangles in Fig. 5 ). As the second moment $\sigma=\left\langle s^{2} P(s)\right\rangle_{\xi}$ of the NNSD decreases with increasing disorder, one could alternatively obtain an estimate for the roughness of a flake from $\sigma$. We find however, that the dependence on $\beta$ is numerically more reliable, as the entire distribution is used for a fit to $P_{H}$.

While the shape of the unfolded NNSD, $P(s)$, is sensitively dependent on disorder, it is to a good degree of approximation size independent, as our numerical data confirms (not shown). The reason for this is the normalization $\langle s P(s)\rangle=1$, which scales out size-related effects.
By contrast, the absolute level spacing $\langle\Delta E\rangle$ contains direct information on the size of quantum confinement. By rescaling each level spacing by the local energy, relative to a fixed energy $E_{0}=100 \mathrm{meV}$, one obtains the energy independent expectation value

$$
\bar{\delta}=\left\langle\Delta E \cdot \frac{E}{E_{0}}\right\rangle=\left(\hbar v_{\mathrm{F}}\right)^{2} \frac{2}{d^{2} E_{0}}=\frac{5500 \mathrm{~nm}^{2}}{d^{2}} \mathrm{meV} .
$$

This rescaled mean level spacing is, indeed, independent of edge roughness and disorder [see Fig. 5(b)]. Agreement with the experimental data [6] for $d=40 \mathrm{~nm}$ is surprisingly good.

In conclusion, the spectrum of realistic graphene quantum dots in the presence of disorder (edge roughness or defects) reveal unique features which differ from both Schrödinger or Dirac billiards of confined massive or massless free particles. The graphene bandstructure near the $K$ point leaves clear imprints. They include interference structures in the wavefunctions, enhanced confinement effects, and a delayed transition from Poisson to Wigner-Dyson nearest-neighbor distributions. While one still "cannot hear the imperfect shape of the drum", the size and roughness of graphene quantum dots can be, indeed, inferred from the spectral properties.

We thank K. Ensslin, T. Ihn, S. Rotter and L. Wirtz for valuable discussions. F.L. and J.B. acknowledge support from the FWF-SFB Adlis, C.S. support from NCCR.

[1] K. S. Novoselov et al., Nature, 438, 197-200, (2005).

[2] Y. Zhang, Y.-W. Tan, H. L. Stormer, P. Kim, Nature, 438, 201-204, (2005).

[3] A. K. Geim and K. S. Novoselov, Nat. Mater. 6, 18 (2007)

[4] B. Trauzettel, D.V. Bulaev, D. Loss, and G. Burkard, Nature Physics, 3, 192, (2007).

[5] M. V. Berry, F. R. S. and R. J. Mondragon, Proc. R. Soc. Lond. A 412, 53-74 (1987).

[6] L. A. Ponomarenko et al., Science, 320, 356 (2008).

[7] H. Min et al., Phys. Rev. B, 74, 165310, (2006).

[8] N. Tombros et al., Nature, 448, 571-574, (2007).

[9] M. I. Katsnelson, K. S. Novoselov, amd A. K. Geim, Nature Phys. 2, 620 (2006).

[10] N. Dombay, and A. Calogeracos, Phys. Rep., 315, 4158 (1999)

[11] M. Y. Han, B. Özyilmaz, Y. Zhang, and P. Kim, Phys. Rev. Lett., 98, 206805 (2007).

[12] Z. Chen, Y. Lin, M. Rooks and P. Avouris, Physica E, 40, 228, (2007).

[13] Y.-M. Lin, V. Perebeinos, Z. Chen, and P. Avouris, arXiv:0805.0035 $v 2$ (2008)

[14] F. Miao, S. Wijeratne, Y. Zhang, U. C. Coskun, W. Bao, C. N. Lau, Science, 317, 1530 (2007)

[15] C. Stampfer et al., Appl. Phys. Lett., 92, 012102 (2008).

[16] C. Stampfer, E. Schurtenberger, F. Molitor, J. Güttinger, T. Ihn, and K. Ensslin, Nano Lett., in press (2008).

[17] J.-H. Chen et al.,Nature physics, 4, 377 (2008)

[18] C. Lanczos, Journal of Research of the National Bureau of Standards, 45, 255, (1950) 
[19] S. Reich, J. Maultzsch, C. Thomsen, and P. Ordejon, PRB 66, 035412 (2002)

[20] Y.-W. Son, M. L. Cohen, and S. G. Louie, PRL 97, 216803, (2006).

[21] N. M. R. Peres, A. H. CastroNeto, and F. Guinea, Phys Rev. B 73, 195411, (2006) D. Dunlycke, D. A. Areshkin, and C. T. White, Appl. Phys. Lett. 90, 142104, (2007) J. Fernandez-Rossier, J. J. Palacios, and L. Brey, Phys. Rev. B 75, 205441, (2007)
[22] M. V. Berry and M. Robnik, J. Phys. A 17, 2413 (1984).

[23] T. A. Brody, Lett. Nuov. Cim. 7, 482 (1973).

[24] E. Caurier, B. Grammaticos, and A. Ramani, J. Phys. A 23, 4903 (1990).

[25] F. M. Izrailev, Phys. Lett. A134, 13 (1988).

[26] G. Lenz and F. Haake, Phys. Rev. Lett. 67, 1 (1991).

[27] H. Hasegawa, H. J. Mikeska and H. Frahm, PRA 38, 395 (1988). 